\title{
Analysis of Photocarrier Dynamics at Interfaces in Perovskite Solar Cells by Time-Resolved Photoluminescence
}

Ahmer A.B. Baloch, Fahhad H. Alharbi, Giuilia Grancini, Mohammad I. Hossain, Md. K. Nazeeruddin,

Nouar Tabet

\begin{abstract}
The power conversion efficiency of perovskite solar cells is drastically affected by the carrier dynamics at the interfaces. Experimental measurements under continuous excitation show a quenching of the photoluminescence (CW-PL) signal from the perovskite layer when it is capped with a hole transport medium (HTM). Furthermore, time resolved photoluminescence (TRPL) data show a faster decay of the PL signal in presence of perovskite/HTM interface. The experimental decay is usually fitted using one or two exponential functions with incomplete physical picture. In this work, we calculate the decay of the PL signal in presence of both defect assisted recombination (SRH) and band-to-band radiative recombination. The carrier extraction/recombination at the interfaces is described by the interface recombination velocity. The proposed model captures the temporal and spatial distribution of TRPL signal by fitting the experimental data and extracting the key physical parameters characterizing the carrier dynamics in the bulk as well as at the interfaces of the studied films. Notably, the inclusion of diffusion, doping density and surface recombination velocity at interfaces allow to accurately capture the physics of TRPL kinetics by improving the conformity of fits to experiments. This method of deconvoluting physical parameters from standard TRPL measurements can assist engineers and scientists to assess and improve the device parameters and process techniques.
\end{abstract}

\section{Introduction}

Since their discovery, perovskite solar cells (PSC) unleashed a great deal of enthusiasm in the PV community because of their technological promises and the open questions raised concerning the physical processes occurring in the device. The nature of the gap (direct/indirect) ${ }^{1-3}$, the values of the carrier mobilities and diffusion length ${ }^{4-6}$,the charge transport mechanism ${ }^{7-11}$, the role of point defects ${ }^{12,13}$, and the recombination mechanisms responsible of the carrier losses ${ }^{14-18}$ have been the subject of intensive debates. In the standard configuration, a PSC consists of a thin perovskite absorbing layer sandwiched between an electron transport medium (ETM) and a hole transport medium (HTM). Under light excitation, the photocarriers are generated in the perovskite absorbing layer and diffuse towards the interfaces. Because of the appropriate band alignment at the interfaces, electrons are extracted at the ETM/perovskite interface while holes are extracted at the perovskite /HTM interface ${ }^{19}$. The carrier extraction at these interfaces occurs within picosecond range after excitation $^{20,21}$. The radiative band to band recombination leading to Photoluminescence (PL) occurs in the nanosecond range ${ }^{22,23}$. The photocarriers generated close to the interfaces are extracted before contributing to the PL signal leading to the PL quenching. The drastic reduction of PL signal in presence of HTM /perovskite interface has been assigned by many authors to carrier extraction at the selective electrodes ${ }^{24}$.

Time resolved Photoluminescence (TRPL) has been used extensively to investigate the carrier extraction at the interfaces of perovskite solar cells ${ }^{9,25-29}$. The contactless nature and its application for both complete and noncomplete device makes it an ideal tool for characterizing limitations in PSC. Careful physical examination of TRPL measurements can provide us with an understanding of the complex attributes of charge dynamics and extraction mechanism in mixed halide PSC. In a typical TRPL experiment, the sample is excited by a very short laser pulse leading to the generation of an initial excess carrier density $n(0)$ which subsequently decays as a 
result of the recombination and carrier extraction at the interfaces as illustrated by Figure 1a. Figure 1 shows the TRPL schematic along with conventional curve fitting and charge dynamics fitting method for PL measurements. Conventionally, TRPL curves are analyzed by performing curve-fitting to the PL transients and extracting the associated lifetimes. Due to the complex quasi-exponential shape of the TRPL; one exponent, two exponent and stretched exponential functions for fitting have often been employed as shown in Figure $1 \mathrm{~b}$. The main limitation arising from this method is the extraction of arbitrary life time constants without consistent consideration of the physical processes involved in TRPL decay dynamics. Moreover, as the diffusion and varied recombination pathways in bulk are occurring at comparable time scales, there is a high likelihood that the extracted parameters may be convoluted with other properties. To understand the opto-electronic properties and obtain meaningful insights, charge transport for analyzing PL transients must be taken into consideration. This assists in unraveling the physical factors governing the PL decay under different time scale and conditions.

The determination of the PL decay requires solving the continuity equation taking into account the generation, transport, recombination and extraction of the carriers. ${ }^{29-31}$. In the practical experimental setup, the incident light penetrates into the cell through the transparent glass, FTO and ETM layers and gets absorbed exponentially in the perovskite layer according to Lambert law. Therefore, a diffusion term needs to be included in the continuity equation which governs the charge dynamics after a pulsed excitation. The effect of doping concentration, intentional or unintentional, must be considered as well to study the collective impact of doping and radiative recombination on TRPL kinetics ${ }^{8,32}$. More importantly, the addition of surface recombination velocity and diffusion provides detailed insight of the complex kinetics occurring at initial PL decay. This can equip us with the true quality of absorbers coupled with HTM or ETM. We describe in this work, such approach along with the boundary conditions and optimization algorithm to consider the carrier extraction or/and recombination at the interfaces. This fundamental work can benefit in understanding the details of the transport and extraction kinetics by dismembering the effect of individual parameters contributing to TRPL in PSC. By coupling this comprehensive model with the experimental measurements, the aim is to go beyond the conventional fitting parameters of life times and extract the parameters with physical significance such as surface recombination/interface recombination velocity $\left(S_{L}\right.$ and $S_{R}$ ), monomolecular recombination constant $\left(k_{1}\right)$, bi-molecular recombination constant $\left(k_{2}\right)$, mobility $(\mu)$ and doping density $\left(N_{D}\right)$ as shown in Figure $1 \mathrm{c}$. 
a) TRPL measurements

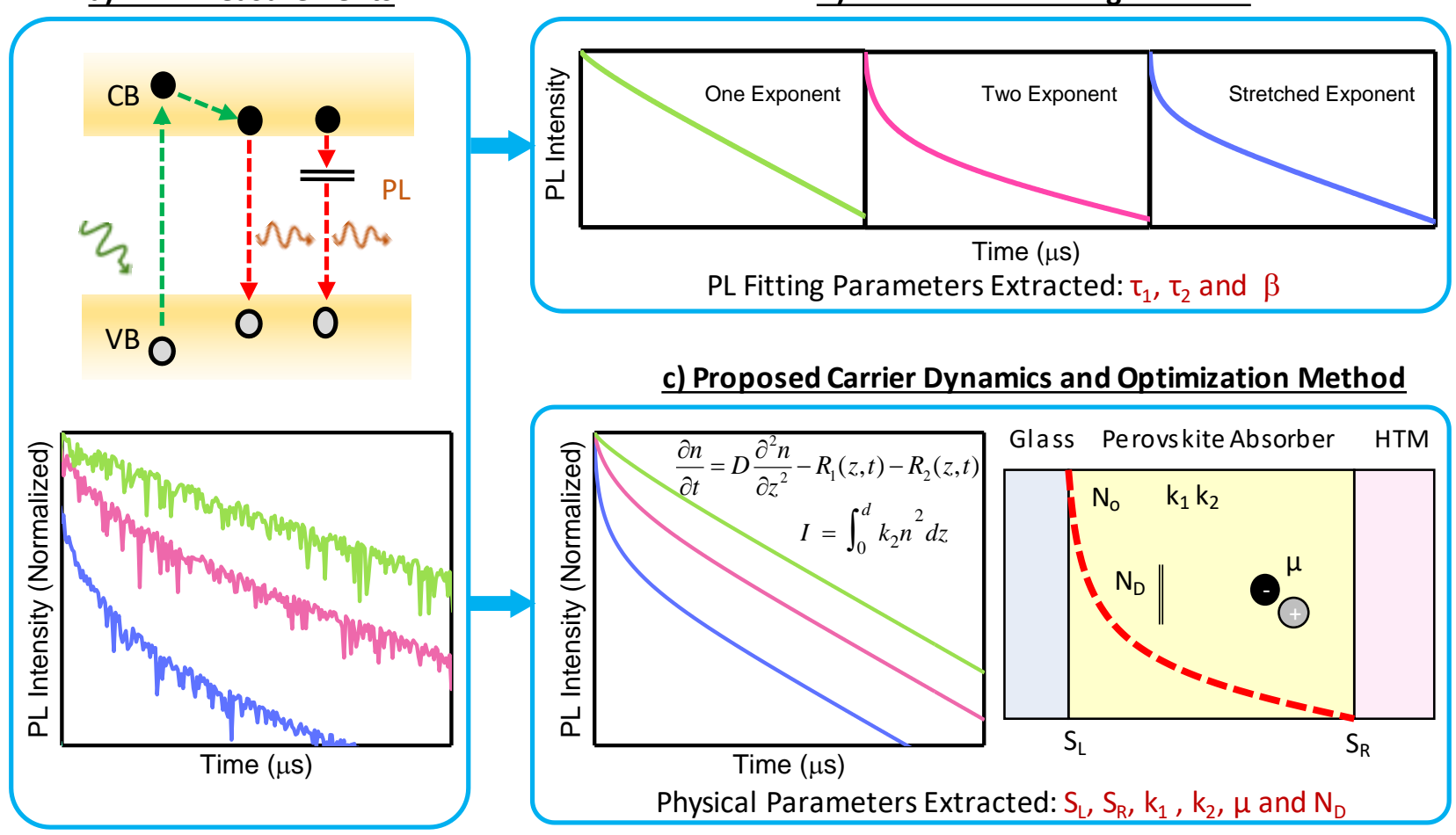

Figure 1 a) TRPL signals arising from recombination pathways b) Conventional fitting method of TRPL measurements for fitting constants. PL curves are often fitted by one, two and stretched exponential functions. Here $\tau_{1}, \tau_{2}$ and $\beta$ show lifetimes and stretched constant, respectively. c) The proposed charge dynamics method predicts physical parameters by solving rate equation with appropriate boundary conditions based on the geometry. By numerical optimization, it deduces the opto-electronic properties from measurements. Here, surface recombination/interface recombination velocity $\left(S_{L}\right.$ and $\left.S_{R}\right)$, monomolecular recombination constant $\left(k_{1}\right)$, bi-molecular recombination constant $\left(k_{2}\right)$, mobility $(\mu)$ and doping density $\left(N_{D}\right)$ are the physical parameters extracted.

\section{Time Resolved Photoluminescence modeling}

Let us consider the case of a perovskite layer deposited on a glass substrate and covered by a thin HTM layer as displayed in Fig. 2 a) and 2b).

The continuity equation in the perovskite layer can be written on the following form:

$$
\frac{\partial n}{\partial t}=D \frac{\partial^{2} n}{\partial z^{2}}-R_{1}(z, t)-R_{2}(z, t)
$$

where $n$ is the minority excess carrier density, $D$ is the carrier diffusion constant, $z$ is the distance from the origin taken at the location of the interface HTL/perovskite, $R_{I}$ is the recombination constant associated to Shockley Read Hall (SRH) recombination, and $R_{2}$ is the recombination rate associated with the band to band recombination (bimolecular process). The Auger recombination is negligible under the normal working condition of solar cells (below an excess carrier density of $10^{17} \mathrm{~cm}^{-3}$ ) 33 . The end of the excitation laser pulse is taken as the time origin (i.e. at $t=0$ ). 
The SRH recombination rate is a function of the photocarrier density, the energy level of the trap $\left(E_{t}\right)$, its cross section $\sigma$ and density $N_{t}{ }^{34}$.

$$
R_{1}=\frac{\left(n_{0}+n\right)\left(p_{0}+p\right)-n_{i}^{2}}{\left(n_{0}+n\right)+\left(p_{0}+p\right)+2 n_{i} \cosh \left(\frac{E_{i}-E_{t}}{k T}\right)} \sigma v_{t h} N_{t}
$$

Where $n_{0}$ and $p_{0}$ are the equilibrium carrier densities of electrons and holes respectively, $n$ and $p$ are the densities of photogenerated carriers $(n=p), E_{i}$ is the mid gap and $E_{t}$ is the trap energy level.

Under low injection level conditions, the photocarrier density $n$ is much lower than the equilibrium density of the majority carriers, $R_{1}$ can be expressed as linear function of the photocarrier density $n(z, t)$

$$
R_{1}(z, t) \approx k_{1} n(z, t)
$$

where $k_{1}$ is the SRH recombination frequency. For $n$-type absorber, $k_{1}$ is given by:

$$
k_{1}=\frac{\sigma v_{t h} N_{t}}{1+2 \frac{n_{i}}{n} \cosh \left(\frac{E_{i}-E_{t}}{k T}\right)}
$$

$R_{2}$ is given by :

$$
R_{2}(z, t)=k_{2}\left[\left(n_{0}+n\right)\left(p_{0}+p\right) \quad n_{i}^{2}\right]
$$

where $k_{2}$ is the band-to-band recombination constant which is a characteristics of the material. It is clear from Eq. 2 that $R_{1}$ has some radiative part as in Eq. (5).

Assuming $n$-type absorber, and considering the low injection condition. $n=p \ll n_{0}=N_{D}$, the term $R_{2}$ becomes also a linear function of the photocarrier density:

$$
R_{2}(z, t) \approx k_{2} N_{D} n
$$

Therefore Eq. (1) can be rewritten under low injection conditions as follows:

$$
\frac{\partial n}{\partial t}=D \frac{\partial^{2} n}{\partial z^{2}}-\left(k_{1}+k_{2} N_{D}\right) n
$$


Notice that the term $k_{2} N_{D}$ is non negligible only if the absorbing layer is highly doped (for typical values of $k_{1}=10^{7} \mathrm{~s}^{-1}$ and $\left.k_{2}=10^{-10} \mathrm{~cm}^{3} \mathrm{~s}^{-1}, N_{D}>10^{17} \mathrm{~cm}^{-3}\right)$. Therefore, under low injection level conditions, and low doping concentration, the decay of the photogenerated carriers is mainly controlled by the SRH recombination rate $k_{1}$.

Under high injection levels, Eq. (1) takes the following form

$$
\frac{\partial n}{\partial t}=D \frac{\partial^{2} n}{\partial z^{2}}-\left(k_{1}^{\prime}+k_{2} N_{D}\right) n-k_{2} n^{2}
$$

where $k_{1}^{\prime}$ is given by

$$
k_{1}^{\prime}=\frac{\sigma v_{t h} N_{t}}{2\left\{1+\frac{n_{i}}{n} \cosh \left(\frac{E_{i}-E_{t}}{k T}\right)\right\}}
$$

Note that the variation of $k_{1}^{\prime}$ on the injection level depends upon the level of the trap. For deep level, that is $E_{i}-E_{t} \gg k T, k_{1}^{\prime}$ is half of its value under low injection conditions, that is

$$
k_{1}^{\prime}=\frac{\sigma v_{t h} N_{t}}{2}
$$

However, for shallow levels, $k_{1}^{\prime}$ increases as the density of photocarriers $n$ increases.

As shallow level contributes very little to the recombination process under low injection levels, we will consider only the deep levels in our analysis. Therefore, in the presence of deep levels, Eq. (1) can be rewritten in the following form for all injection levels:

$$
\frac{\partial n}{\partial t}=D \frac{\partial^{2} n}{\partial z^{2}}-\left(k_{1}^{\prime}+k_{2} N_{D}\right) n-k_{2} n^{2}
$$

Where the constant $k_{1}^{\prime}$ varies from $k_{1}$ to $\frac{k_{1}}{2}$ as the injection is increased from low to high levels.

Considering the experimental configuration shown in Fig. 2, the photoluminescence signal is then obtained by integrating the radiative term over the absorber as follows

$$
I_{P L}=\int_{0}^{d} k_{2} n^{2} d z
$$


In order to obtain the PL signal decay versus time, we need to specify the boundary conditions of the problem and determine the function $n(z, t)$ by solving Eq. (11). These boundary conditions can be expressed at the ends of the absorbing materials by introducing a surface /interface recombination velocity, $S$. The inclusion of surface recombination velocity along with diffusion term provides an accurate assessment of the complicated kinetics at the initial PL signal.

In the case of an absorbing layer deposited on glass:

$$
J(0, t)=S_{L} n(0, t)
$$

and

$$
J(d, t)=S_{R} n(d, t)
$$

where $J$ is the carrier flux and $S$ is the surface/interface recombination velocity.

Note that, in absence of HTM layer (Fig. 2a), $S_{R}$ characterizes the surface recombination at the free surface of the absorber. However, in presence of HTM layer (Fig. 2b), $S_{R}$ integrates both the carrier extraction from the absorber to the HTM layer as well as a possible SRH recombination due to defects at the interface. $S_{L}$ highlights the recombination velocity at the excited side of the substrate/sample film.

Radiative Efficiency or Photoluminescence Efficiency (PLE) provides an upper limit for solar cell where the main motivation is to decrease the trap assisted recombination ${ }^{35}$. It is a fraction of the radiative recombination to the total radiative and non-radiative SRH recombination ${ }^{36}$. Since this is a measure of emission and absorption of photons, PLE provides a direct figure of merit of material quality by linking the parameters such as $N_{0}, k_{1}$, $k_{2}$ and $N_{D}$ as given by Eq. (13). Care must be taken when comparing PLE as it is governed by nature of the bandgap, direct or indirect.

$$
P L E=\frac{k_{2}\left(N_{D}+n\right)}{k_{1}+k_{2}\left(N_{D}+n\right)}
$$

The initial distribution of carriers is given by

$$
n\left(z, t_{0}\right)=N_{0} e^{(\alpha z)}
$$

where $\alpha$ is the linear coefficient of absorption of the absorbing material and $N_{0}$ is the initial photocarrier concentration at the glass/absorber interface.

The rate equation, Eq. (11), can be solved numerically to determine the carrier distribution $n(z, t)$. Considering the stiff nature of the problem, an implicit solver must be used.

Two observations can be pointed out: 
1) The effect of carrier extraction is expected to becomes measurable only when the carrier diffusion length $L=\sqrt{D \tau}$ is comparable or larger than the thickness $(d)$ of the absorbing layer.

2) The extraction of the carriers at the interface may lead to a charge build up at the interface which, in return, would affect the carrier extraction thus the parameter, $S$. Such possible effect would induce more complexity in the problem and is neglected in the current analysis. 
a)

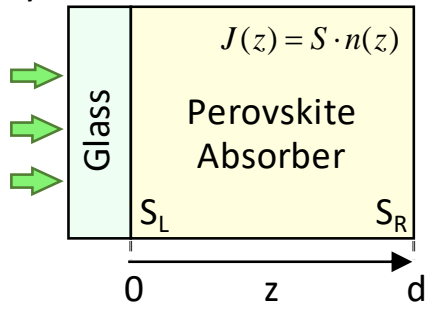

b)

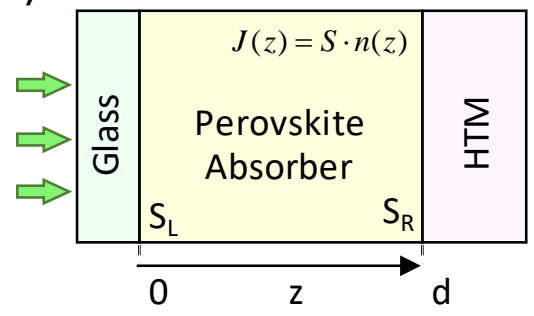

c)

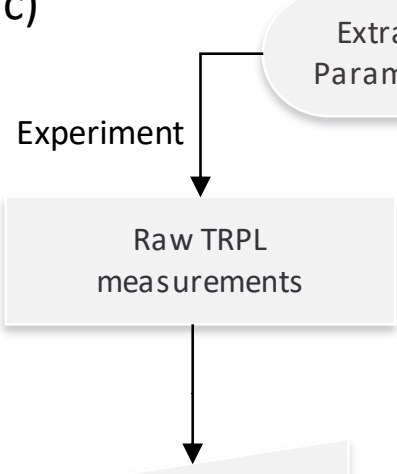

Extracting Physical

arameters from TRPL

Modeling

Charge dynamics Model

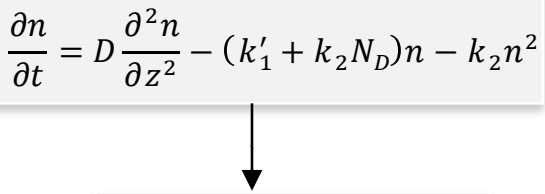

Smooth data and

Boundary Conditions

$J(0, t)=S_{L} n(0, t)$

reduce noise, $I_{P L, e}$

$J(d, t)=S_{R} n(d, t)$

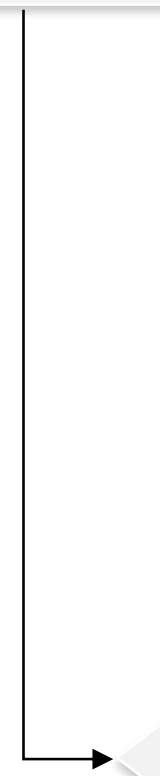

$E=\left\|I_{P L, e}-I_{P L, m}\right\|$

Not Converged

Criteria: $\mathrm{E}<10^{-6}$

Converged

for optimizing parameters

$S_{L}, S_{R}, k_{1}, k_{2}, \mu$ and $N_{D}$

Numerically solve TRPL intensity

$$
I_{P L, m}=\int_{0}^{d} k_{2} n^{2} d z
$$

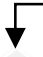

Extracted Parameters

$S_{L}, S_{R}, k_{1}, k_{2}, \mu$ and $N_{D}$

Figure 2 Schematic for samples and methodology for TRPL analysis for PSC. a) Perovskite deposited on glass (no carrier extraction), b) Glass structure/Perovskite/HTM with carrier extraction at Perovskite/HTL interface. c) Flow chart 
describing the TRPL numerical modeling and charge dynamics based optimization method. The proposed solver is capable for simultaneously extracting physical parameters from device such as $S_{L}, S_{R}, k_{1}, k_{2}, \mu, N_{D}$.

\section{Numerical Methodology}

Figure $2 \mathrm{c}$ shows the flowchart for the numerical modeling and charge dynamics based optimization method. The experimentally obtained normalized TRPL signal, $I_{P L} / I_{P L, \max }$, is smoothened first by reducing the machine associated noise. Poor signal-to-noise ratio associated with long decay tails with weak PL emission may hinder proper extraction of $n(t)$ resulting in unreliable parameters. In addition, care must be taken while selecting the time scale for the PL decay to be considered as this may overestimate or underestimate the processes occurring at different time regimes such as trap assisted recombination $k_{1}$ at longer time scales and radiative recombination $k_{2}$ at short time scale. For the extraction of parameters from TRPL curves, a gradient based multi-start optimization algorithm was developed and employed for an objective function of error between measured PL and modeled PL decay as given by Eq. (15).

$$
\left\|E_{P L}\right\|=\left\|I_{P L, \text { measured }}(z, t)-I_{P L, \text { modeled }}\left(S_{L}, S_{R}, k_{1}, k_{2}, \mu, N_{D}\right)\right\|
$$

where $\|\cdot\|$ is the $2^{\text {nd }}$ norm.

The numerically modeled PL decay is capable of tracing temporal variation of optoelectronic properties along with the transport of minority carriers spatially. The collective reconstruction of spatial and temporal profile of PL makes it a robust model for precise determination of surface recombination ( $S_{L}$ and $S_{R}$ ), SRH recombination frequency $\left(k_{1}\right)$, Band-to-band radiative recombination $\left(k_{2}\right)$, carrier mobility $(\mu)$ and doping density $\left(N_{D}\right)$. Numerically, higher order finite difference based scheme with Neumann boundary condition for surface recombination is employed for spatial discretization and the resulted rate equation of the system is solved using fully implicit ODE solver for calculating the PL signal in Eq. (12). Shorter time steps are used at the early time regimes to effectively capture the charge carrier physics with step size $\left(\sim 10^{-12}\right)$. The fitting procedure is provided with an initial vector for optimizing parameters of $S_{L}, S_{R}, k_{1}, k_{2}, \mu, N_{D}$ and is iteratively solved until the stopping criteria of $\left\|E_{P L}\right\|<10^{-6}$ between experimental and PL modeled decay is satisfied.

\section{Results and Discussion}

\subsection{TRPL modeling analysis}

In the following section, we generate a series of plots showing the effect of various physical parameters such as $S_{L}, S_{R}, k_{1}, k_{2}, \mu, N_{D}, N_{0}$ on the PL signal decay. This enables us to assess the sensitivity of the curves to these parameter changes and the possibility to extract these parameters from the experiment using an optimization fitting procedure. Then we will illustrate the methodology by using it for the analysis of PL curves obtained on perovskite thin films (sample on glass with and without an HTM layer) and single crystals.

Figure 3 shows typical PL curves for various values of surface recombination velocity: $S_{L}$ and $S_{R}$, where $S_{L}$ is at the glass, ETM or FTO interface and $S_{R}$ is at the interface with HTM. Qualitatively, surface recombination 
velocity, at interface results in quenching of PL decay showing a reduction in PL signal ${ }^{37}$. Figure $3 \mathrm{a}$ and $3 \mathrm{~b}$ shows the effect of $S_{R}$ on PL kinetics due to varying recombination at sample/HTM interface. Here, two values for $S_{L}$ are considered $\left(10^{1} \mathrm{~cm} / \mathrm{s}\right.$ and $\left.10^{5} \mathrm{~cm} / \mathrm{s}\right)$. The PL signal quenching is due to the efficient carrier transfer to the interface which may include both carrier extraction from the absorber to HTM and possible SRH recombination due to interfacial defects ${ }^{31}$. Note that, in absence of HTM, $S_{R}$ describes the recombination at the free surface. If the recombination is negligible at the free surface, then the presence of HTM is expected to increase $S_{R}$ due to the extraction process. With higher $S_{L}$, i.e. more extraction from the photo-excited side, the quenching of PL curve is larger as the overall impact of trap assisted recombination is significant. At a longer time scale, both curves follow mono-exponential decay where $k_{1}$ becomes dominant over $k_{2} N_{0}$.

Figure3c shows the effect of $S_{L}$ on the PL signal. The initial decay signifies the carrier quenching to the adjacent glass/absorber interface. It is evident that PL decays more rapidly for injections near the samplesubstrate interface for both $S_{R}$ and $S_{L}$ cases. Therefore, for curve fitting of PL measurements, it is important to decouple both surface recombination velocity and diffusion to assess the quality of each interface with the absorber ${ }^{29}$. The key advantage of analyzing surface recombination is its direct relation with device performance and process parameters for fabricating PSC $^{38}$. This in turn can provide design guidelines for optimal device performance such as indicating the requirements for surface layer passivation ${ }^{39}$. 

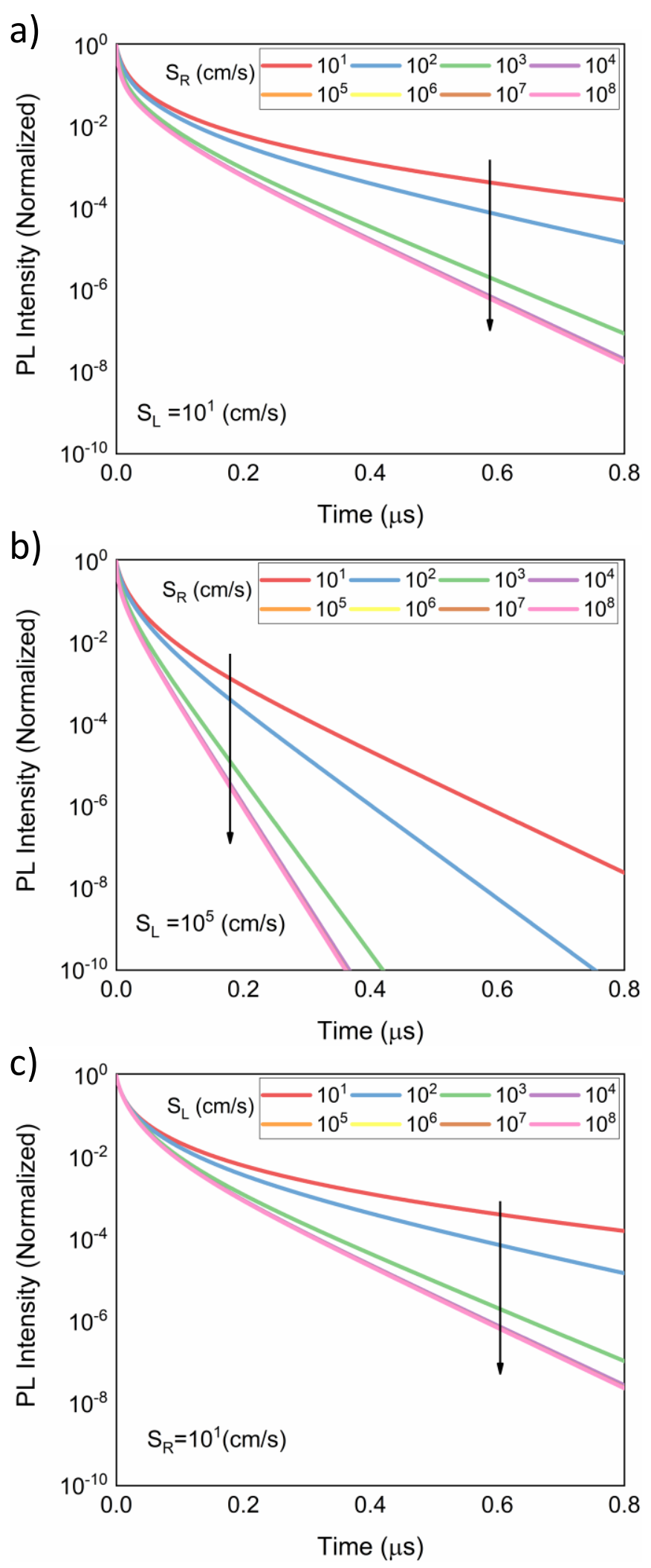
Figure 3 Effect of surface recombination velocity: Calculated surface /interface recombination velocity, $S_{L}$ and $S_{R}$, for perovskite films. Here $S_{L}$ and $S_{R}$ characterizes the surface recombination at the glass/absorber interface and free surface of the absorber, respectively. In the presence of HTM layer, $S_{R}$ integrates both the carrier extraction from the absorber to the HTM layer as well as a possible SRH recombination due to defects at the interface. Calculations are based on $d=0.3 \mu m$, $\mu=0.1 \mathrm{~cm}^{2} / V \mathrm{~s}, k_{1}=10^{6} \mathrm{~s}^{-1}, k_{2}=10^{-12} \mathrm{~cm}^{3} / \mathrm{s}, N_{D}=10^{10} \mathrm{~cm}^{-3}, N_{0}=10^{15} \mathrm{~cm}^{-3}$. Respective $S_{L}$ and $S_{R}$ are indicated in the figures with arrows indicating the increase in parameter.
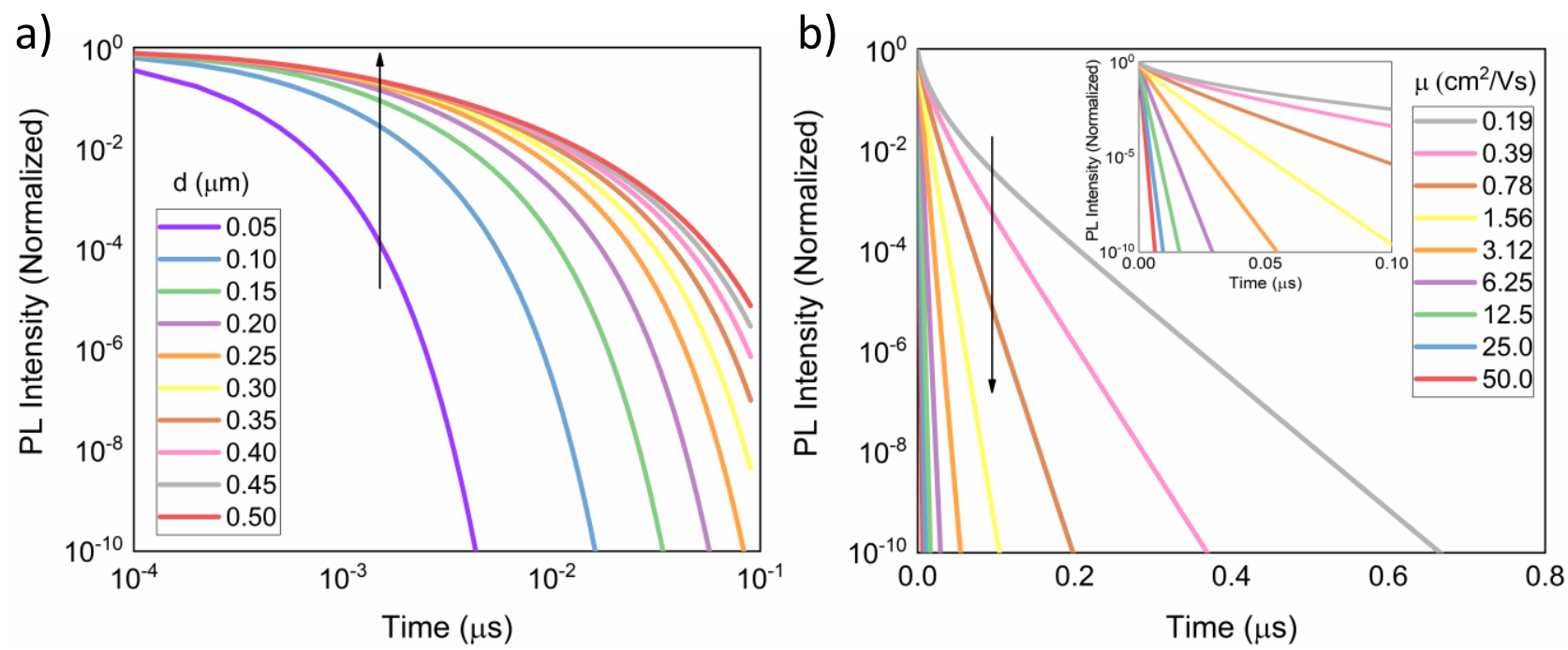

Figure 4 Effect of a) absorber thickness $(d)$ and b) charge carrier mobility $(\mu)$ : Calculations are based on $S_{L}=10^{1} \mathrm{~cm} / \mathrm{s}$, $S_{R}=10^{5} \mathrm{~cm} / \mathrm{s}, k_{l}=10^{6} \mathrm{~s}^{-1}, k_{2}=10^{-12} \mathrm{~cm}^{3} / \mathrm{s}, N_{D}=10^{10} \mathrm{~cm}^{-3}$ and $N_{0}=10^{15} \mathrm{~cm}^{-3}$. Fig 4 a has fixed carrier mobility of $0.1 \mathrm{~cm}^{2} / \mathrm{Ns}$ whereas $4 \mathrm{~b}$ assumes $d=0.3 \mu \mathrm{m}$. The inset in Fig. $4 \mathrm{~b}$ shows the zoomed PL at shorter time scale. The effect of both thickness (d) and minority carrier mobility $(\mu)$ can be better described by carrier diffusion length through $L=\sqrt{D \tau}$ where $D=\frac{k T}{q} \mu$ and $\tau$ represents the effective lifetimes from radiative and non-radiative SRH recombination ${ }^{34}$. Notably, the effect of carrier extraction is significant only when the width $(d)$ of the absorber is comparable or smaller than the diffusion length ( $L \approx 0.5 \mu \mathrm{m}$ in this case with $k_{l}$ as limiting recombination mechanism).

Figure $4 \mathrm{a}$ and $4 \mathrm{~b}$ shows the effect of PL on carrier dynamics as a result of varying thickness and minority carrier mobility. The effect of both thickness $(d)$ and minority carrier mobility $(\mu)$ can be described by carrier diffusion length through $L=\sqrt{D \tau}$ where $D=\frac{k T}{q} \mu$ and $\tau$ represents the effective lifetimes from radiative and non-radiative SRH recombination ${ }^{34}$. Diffusion length, $L$, is the average distance an excess carrier travels before it recombines whereas $\mathrm{D}$ is the diffusion constant of the material at specific temperature $T$. It is dependent on the mobility and life time of the material which characterizes the quality of the material. For a constant mobility of $0.1 \mathrm{~cm}^{2} / \mathrm{Vs}$, increasing the absorber thickness reduces the PL signal as a result of lesser recombination due to the interface shown in Fig.4a . For thinner absorbers, photo-generated charge carries have a higher chance to reach the HTM interface thereby leading to faster PL quenching. Particularly, the effect of carrier extraction is significant only when the diffusion length is comparable or larger than the thickness of the absorber i.e $L \geq d$. By considering SRH to be the dominant recombination mechanism, $k_{1} \gg k_{2}$, diffusion length was found to be $0.5 \mu \mathrm{m}$ in this case. Moreover, combination of the thickness dependency on PL with surface recombination velocity at interface can assist in decoupling the bulk and surface recombination lifetime ${ }^{40,41}$.

Charge carrier mobility $(\mu)$ plays a crucial role in determining the shape of PL signal as displayed in Fig. $4 \mathrm{~b}$. The initial decay of the PL curve is governed by diffusion for the carriers especially for higher injection regime 
where the quadratic dependence of injection on the recombination is significant [Please see Eq. (11)]. Therefore, minority carriers with high mobility diffuses faster to the interface thereby producing a sharper PL decay However, as the carrier mobility decreases, extraction at the interfaces is slowed down due to the possible recombination of the carrier in the bulk resulting in lower PL signal. It should be noted that charge carrier dynamics and consequently the PL signal is found to be extremely sensitive to the carrier mobility especially at the first few $n s$ after excitation ${ }^{42}$.

a)

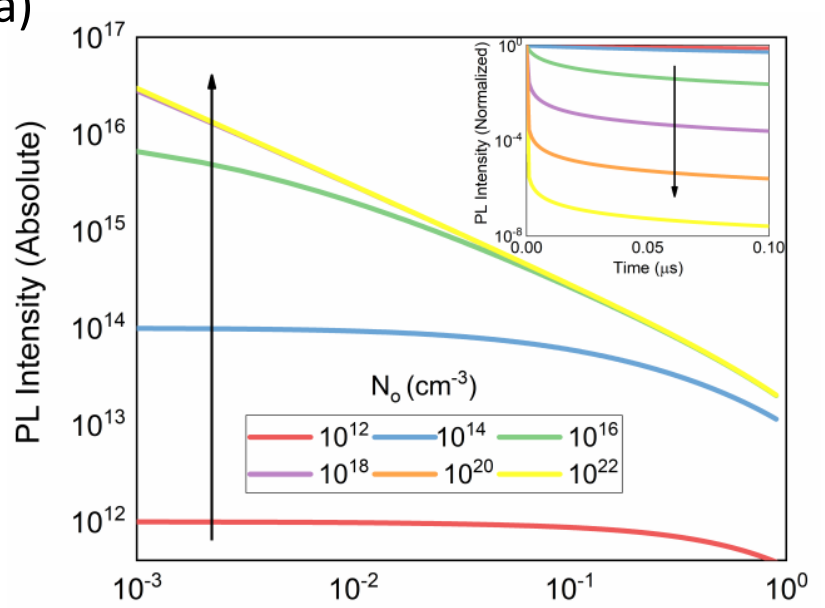

c)

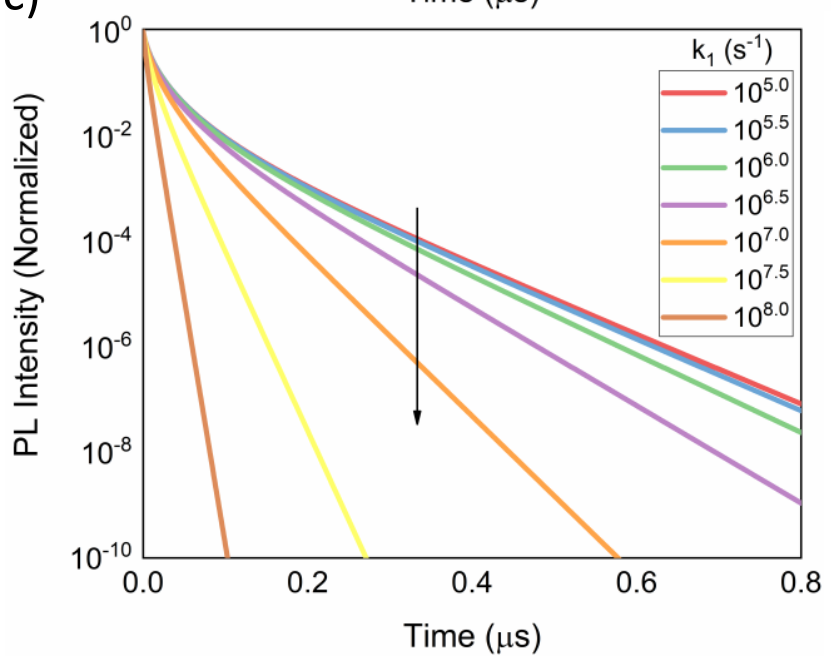

b)
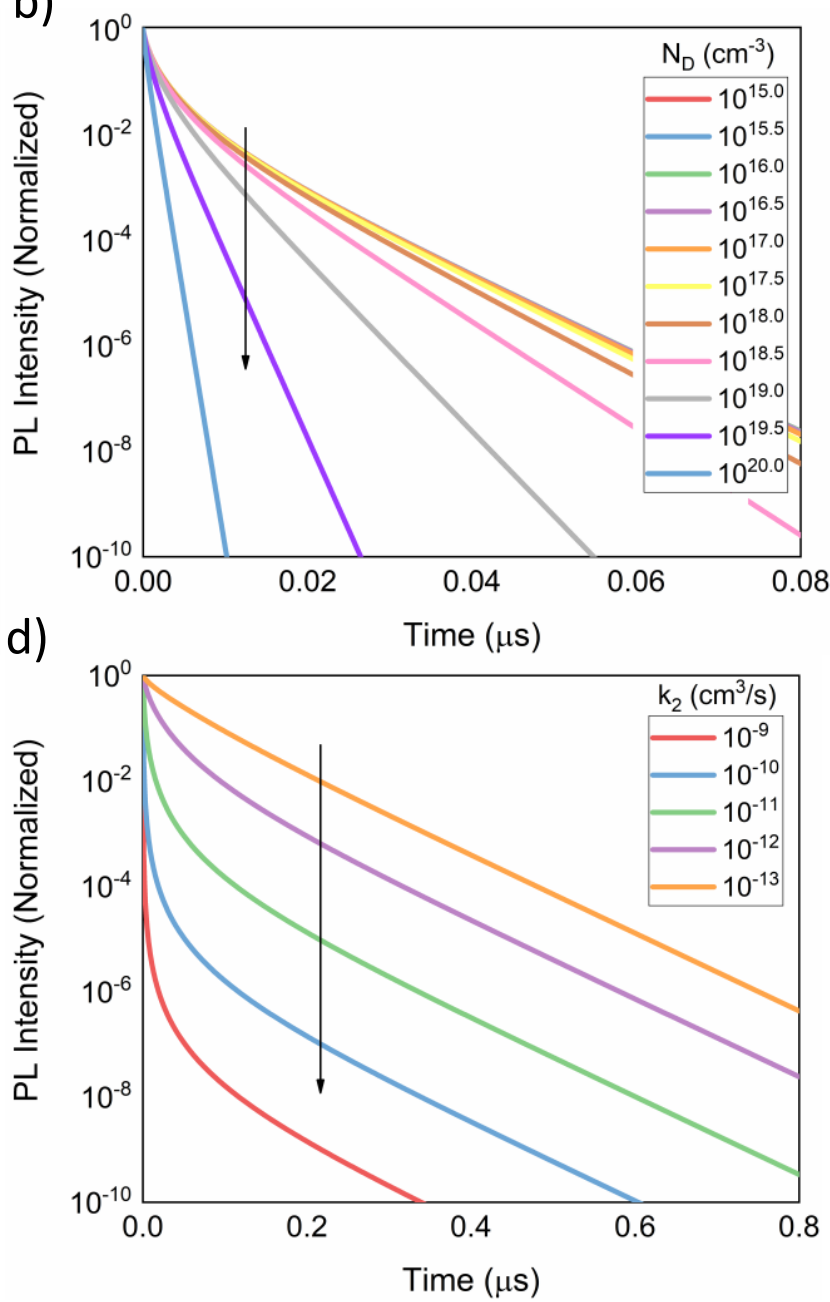

Figure 5 TRPL dependence of initial carrier concentration $\left(N_{0}\right)$, doping density $\left(N_{D}\right)$, SRH recombination frequency $\left(k_{1}\right)$ and Band-to-band recombination $\left(k_{2}\right)$. Calculations are based on $d=0.3 \mu \mathrm{m}, \mu=0.1 \mathrm{~cm}^{2} / V s, S_{L}=10^{1} \mathrm{~cm} / \mathrm{s}$ and $S_{R}=10^{5} \mathrm{~cm} / \mathrm{s}$ with parameters for a) $k_{1}=10^{6} \mathrm{~s}^{-1}, k_{2}=10^{-12} \mathrm{~cm}^{3} / \mathrm{s}, N_{D}=10^{10} \mathrm{~cm}^{-3}$;b) $\left.k_{l}=10^{6} \mathrm{~s}^{-1}, k_{2}=10^{-12} \mathrm{~cm}^{3} / \mathrm{s}, N_{0}=10^{15} \mathrm{~cm}^{-3} ; \mathrm{c}\right) k_{2}=10^{-12} \mathrm{~cm}^{3} / \mathrm{s}$, $N_{D}=10^{10} \mathrm{~cm}^{-3}, N_{0}=10^{15} \mathrm{~cm}^{-3} ;$ d) $k_{l}=10^{6} \mathrm{~s}^{-1}, N_{D}=10^{10} \mathrm{~cm}^{-3}, N_{0}=10^{15} \mathrm{~cm}^{-3}$. The arrows indicate the increase in material parameter analyzed. Fig 5a contains the absolute and normalized (inset) for TRPL decay for better visualization of injection effect.

Figure 5a presents the curves for injection dependent PL kinetics. These TRPL curves can provide a detailed insight into various recombination pathways ${ }^{31,33,36}$. The PL transients and recombination kinetics is a function of injection level, $n(t)$. For better understanding, the effect of injection is discussed in conjunction with $k_{1}$ and 
$k_{2}$. SRH recombination rate, $k_{1}$, signifies the defect assisted recombination in the material. Therefore, from the device and fabrication point of view, the contribution from non-radiative SRH recombination should be lower than its radiative counterpart for a pure material and better crystallinity ${ }^{43}$. In TRPL decays as shown by Fig 5c, SRH follows the mono-exponential decay essentially where the effect of $k_{1}$ becomes dominant over $k_{2} N_{D}$, i.e $k_{1} \gg k_{2} N_{D}$. Under such condition, $n \propto \exp \left(-k_{1}\right)$ and a linear relation is observed at longer time scales. As $k_{1}$ increases, the PL quenching increases due to reduced carrier lifetime and higher recombination. It is important to understand that the variation of SRH recombination upon the injection level depends on the trap level. From shallow to midgap deep level traps (i.e. at $E_{g} / 2$ ), the constant $k_{1}^{\prime}$ varies from $k_{1}^{\prime}$ to $\frac{k_{1}^{\prime}}{2}$ as the injection is increased from low to high. This behavior is caused by the saturation of trap states by the carriers ${ }^{44,45}$. Thus, SRH recombination is a dominant mechanism at low level injection where it is mainly governed by single charge carrier specie. The decay of the photogenerated carriers is mainly controlled by the SRH recombination frequency $k_{1}^{\prime}$ under low injection condition whereas $k_{2}$ is dominant for high level injection (HLI) condition at initial time scale. Physically, higher injection results in more radiative recombination $\left(I_{P L} \propto n^{2}\right.$ ) and carrier extraction at the excited side thereby leading to more quenching at short time scale. Once the carrier moves away from the excited interface, the normalized PL curves results in similar shape with an offset between each other when the effect of $k_{2} N_{0}$ becomes comparable to $k_{1}$. The behavior can be seen clearly from absolute PL curves in Fig. 5a where the effect of injection is proportional to PL signal. One key benefit of using different injection levels is the possibility to acquire better signal-to-noise ratio at larger time scale where the PL emission is weak. This is extremely valuable in capturing the effect of $k_{1}$ from long decay tails ${ }^{37}$.

The effect of doping density ( $N_{D}$ ) on TRPL is an effective measure of a material quality ${ }^{46,47}$. Figure $5 \mathrm{~b}$ shows the effect of doping on the PL kinetics. The transient PL curves at different time scales is either limited by SRH recombination frequency $k_{1}$ or the product of doping density and radiative band to band recombination, $k_{2} N_{D}$ ${ }^{31}$. Generally, the presence of doping, intentional or unintentional, will decrease the carrier life time. The effect of the term $k_{2} N_{D}$ becomes important for a highly doped absorbing layer or very low SRH values (for typical values of $k_{1}=10^{7} \mathrm{~s}^{-1}$ and $k_{2}=10^{-10} \mathrm{~cm}^{3} \mathrm{~s}^{-1}, N_{D}>10^{17} \mathrm{~cm}^{-3}$ ). Consequently, under low injection level (LLI) and low doping concentration, the decay of the photogenerated carriers is mainly controlled by the SRH trap mediated recombination, $k_{1}{ }^{48}$.

Radiative band-to-band recombination, $R_{2}$, is an intrinsic property of the material which characterizes the reciprocal relation between absorption and emission of photon. TRPL is, therefore, a direct measure of radiative recombination. Under LLI $\left(n=p \ll n_{0}\right), R_{2}$ becomes a linear function of the photocarrier density, $R_{2} \propto n(t)$. Contrarily, HLI shows quadratic dependence of recombination rates on the photocarrier density, $R_{2} \propto n(t)^{2}$. Figure 5d shows the effect of variable $k_{2}$ on the PL decay. Under HLI, the effect of $k_{2}$ is dominant which explains the initial decay in the Figure 5b. The effect of $k_{2}$ is dominant when $k_{2} N_{D} \gg k_{1}$. It can be observed that as $k_{2}$ decreases to $10^{-13} \mathrm{~cm}^{3} \mathrm{~s}^{-1}$, the contribution from $k_{1}$ (i.e. mono-exponent behavior) is significantly increased. 


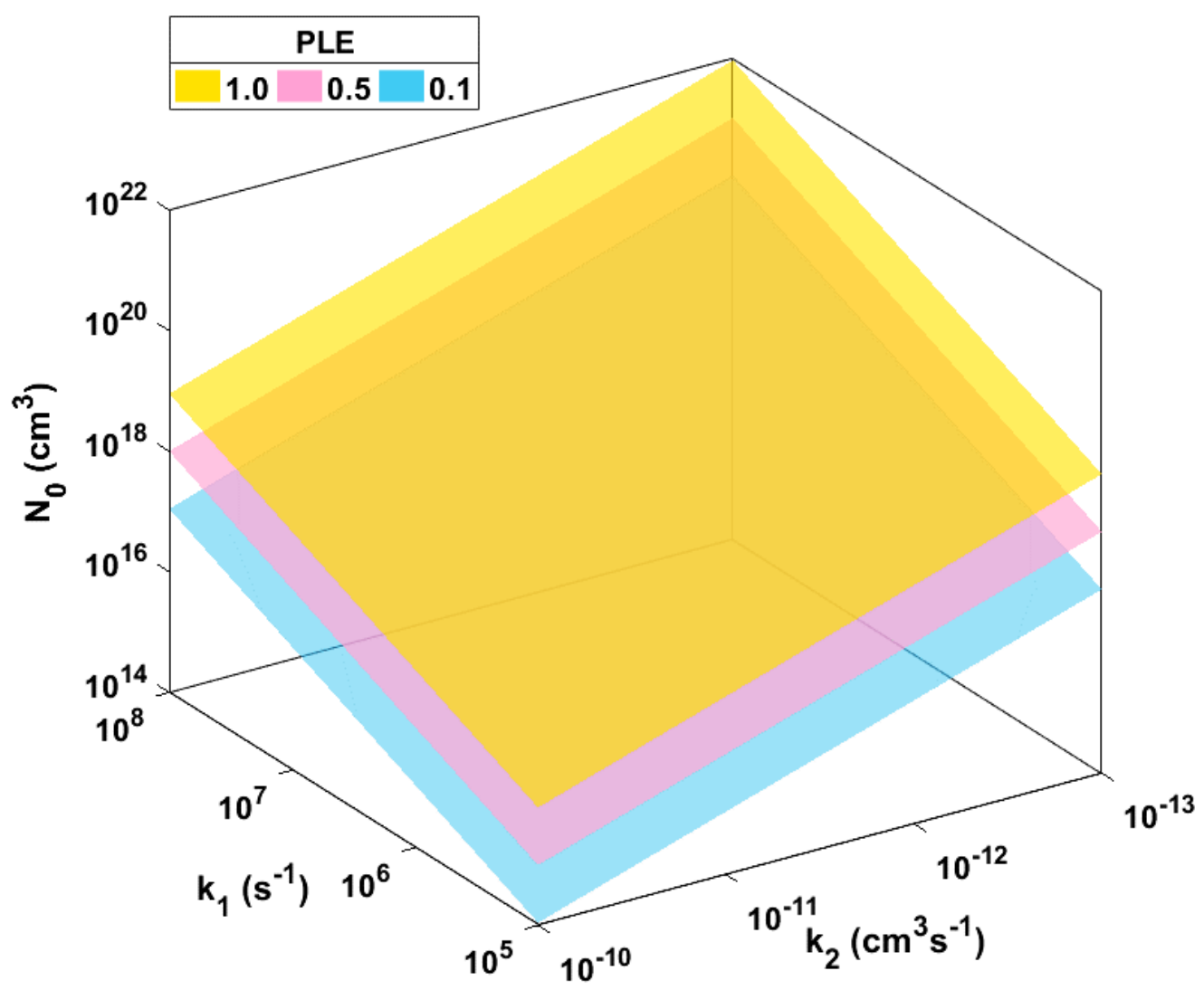

Figure 6 Photoluminescence Efficiency (PLE) trend for injection level $\left(N_{0}\right)$, SRH recombination frequency $\left(k_{l}\right)$ and bandto-band radiative recombination $\left(k_{2}\right)$. The iso-surfaces represent the PLE at specific values of 1.0, 0.5 and 0.1 for different combinations of $k_{1}, k_{2}$ and $N_{0}$ with constant doping density $\left(N_{D}=10^{10} \mathrm{~cm}^{-3}\right)$. PLE is a fraction of the radiative recombination to the total (radiative and non-radiative) recombination.

Figure 6 presents the calculated PLE as function of $k_{1}, k_{2}$, and $N_{0}$ with $N_{D}$ as $10^{10} \mathrm{~cm}^{-3}$. For PLE to be high, $k_{1} \ll k_{2}\left(N_{D}+N_{0}\right)$ under high injection level needs to be satisfied whereas under low injection level this condition reduces to $k_{1} \ll k_{2}\left(N_{D}\right)$. PLE provides an upper limit for solar cell where the motivation is to decrease the trap assisted recombination ${ }^{35}$. However, it should be noted that since PL is a measure of band-toband recombination, PLE is dependent on the nature of band gap of the material. PLE has been used extensively as a performance indicator in light emitting applications such as LED and lasers? 9

\subsection{Analysis of experiment}

Experiments were carried out to analyze the robustness of the developed method and extract physical parameters from TRPL optimization algorithm. For better assessment, thin films (TF) and single crystal (SC) based devices were examined. Thin films for PSC were fabricated with and without HTM whereas single crystal device transients were obtained from Ref. ${ }^{29}$. Figure 7 a) and $7 \mathrm{~b}$ ) shows the PL signal decay obtained on two samples fabricated for thin films. The first one corresponds to a thin film of Methylammonium lead iodide deposited on glass and the second deposited on a similar glass substrate and covered by a thin layer of OMeTAD as hole transport material. TRPL decay for thin films was monitored at excitation powers of $0.466 \mathrm{~mW}, 0.585 \mathrm{~mW}$ and $1.115 \mathrm{~mW}$ with an excitation pulse of $405 \mathrm{~nm}$ and absorber thickness of $0.3 \mu \mathrm{m}$. The fitting is done collectively 
where only $N_{0}$ and $k_{2}$ (to allow for photon recycling) are varied for different excitation levels while all the other parameters are kept common. For single crystal, Fig. 8 shows the measurements which were performed on $10 \mu \mathrm{m}$ thick absorber with an excitation of $447 \mathrm{~nm}^{29}$.

Generally, the type of TRPL decays shown in Fig. 7 and Fig. 8 are fitted by one or two exponential functions as follows ${ }^{49-52}$

$$
I_{P L}=A_{1} \mathrm{e}\left(-\frac{t}{\tau_{1}}\right)+A_{2} \mathrm{e}\left(-\frac{t}{\tau_{2}}\right)
$$

or using a stretched exponential function ${ }^{25-28}$

$$
I_{P L}=A_{1} \mathrm{e}\left(-\frac{t}{\tau_{1}}\right)+A_{2} \mathrm{e}\left(-\frac{t}{\tau_{2}}\right)^{\beta}
$$

The constants $\tau_{1}$ and $\tau_{2}$ are expected to be related to the physical parameters of the absorbing layer such as $k_{1}$, $k_{2}$ and $\mu$ but also to the photocarrier extraction and /or recombination at the interfaces. However, this relation is not straightforward. Here, we show that the physical parameters of the problem such as $k_{1}, k_{2}$ and the interface recombination velocity, $S$, can be extracted by fitting the experimental data to thin films with and without HTM and Single Crystal of perovskite

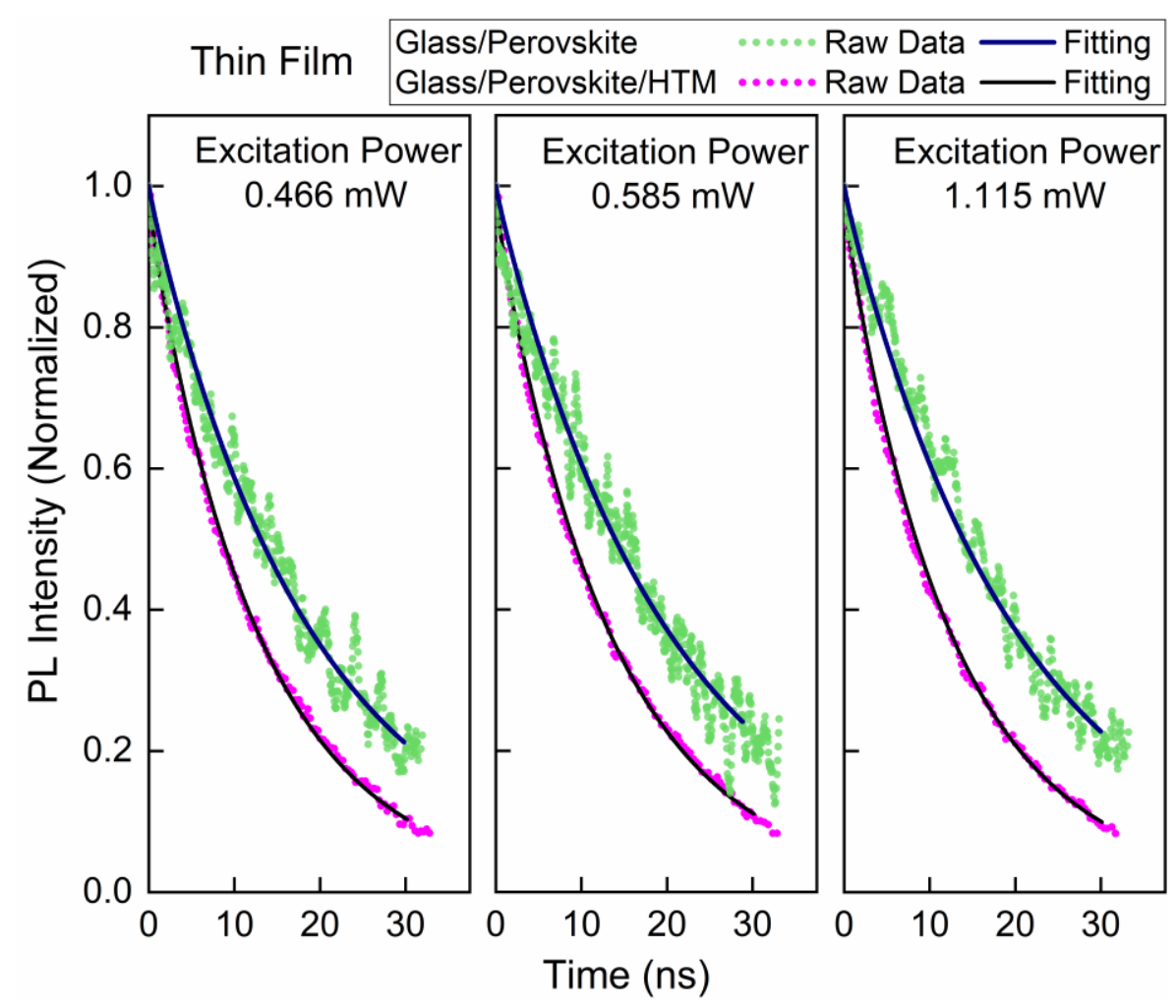


Figure 7 Evaluation of the TRPL fitting on the experimental measurements for perovskite thin films. Samples prepared were Glass/Perovskite and Glass/Perovskite/HTM with sample thickness (d) of $0.3 \mu \mathrm{m}$. Three measurements at excitation power of $0.466 \mathrm{~mW}, 0.585 \mathrm{~mW}$ and $1.115 \mathrm{~mW}$ were carried out with an excitation pulse of $405 \mathrm{~nm}$ and beam size of 800 $\mu m^{2}$.

Table 1 shows the resulted fitting parameters from the proposed charge dynamics and optimization method. Importantly, the inclusion of $\mu, N_{D}, S_{L}$ and $S_{R}$ at both interfaces allowed to accurately capture the physics of TRPL kinetics by improving the conformity of fits to experimental measurements. The decay was found to be higher for thin films in Glass/perovskite/HTM due to the higher rate of extraction at the HTM interface than pure perovskite film. This is also substantiated by the increase in surface recombination velocity from $5.89 \times 10^{4}$ to $8.91 \times 10^{5} \mathrm{~cm} \mathrm{~s}^{-1}$ when we employ HTM for quenching. Interestingly, $S_{R}$ estimated here for thin films is in the same order of magnitude for several unpassivated optoelectronic materials such as $\mathrm{GaAs}\left(\sim 10^{5} \mathrm{~cm} \mathrm{~s}\right)^{-1}$ and n-type $\mathrm{Si}\left(\sim 10^{6} \mathrm{~cm} \mathrm{~s}^{-1}\right)^{54}$. The higher surface recombination velocity, therefore, advocates the usage of surface passivation techniques for PSC ${ }^{55}$. The initial time decay component is also affected by the surface recombination from the glass/absorber interface i.e. the excited side $S_{L}$. It was found to be lower around 24.54 and $5.75 \mathrm{~cm} \mathrm{~s}^{-1}$ for TF and SC, respectively. This can be attributed to the trap filling process due to carrier injection from the excited interface leading to lower interfacial recombination.

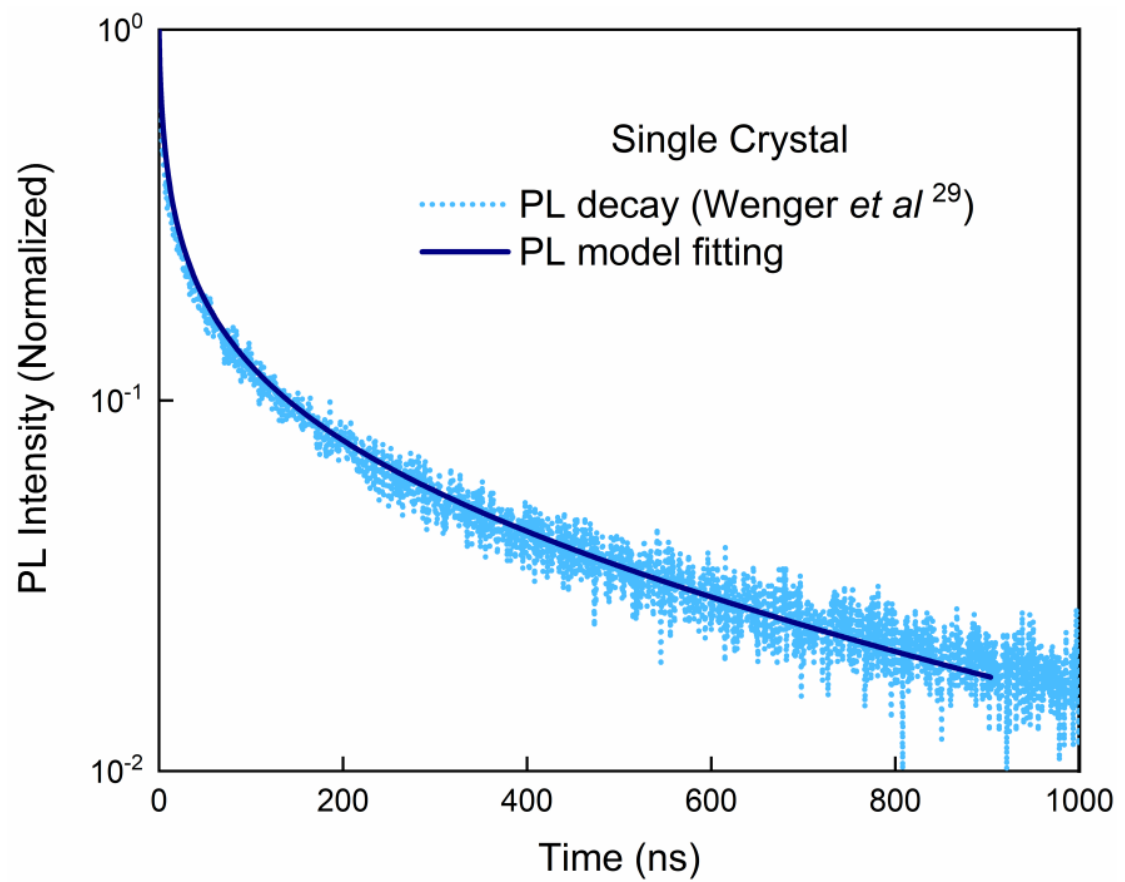

Figure 8 TRPL curve fitting for perovskite single crystal data $\left(10 \mu \mathrm{m}\right.$ thick) obtained from Ref: Wenger et al ${ }^{29}$. Excitation was carried out at $447 \mathrm{~nm}$ with $N_{0}=7.8 \times 10^{16} \mathrm{~cm}^{-3}$.

SC showed a long decay tail up to $1000 n s$ whereas TF exhibited shorter PL decay lasting up to $30 n s$. Qualitatively, this short decay in TF implies that the defect concentration is higher in TF than SC. In Fig. 8 after $1000 \mathrm{~ns}$, the signal to noise ratio was found to be poor for suitable extraction of SRH based parameter. The SRH recombination rate, $k_{1}$, was found to be $7.47 \times 10^{6} s^{-1}\left(\tau_{T F, S R H}=134 n s\right)$ for thin film whereas in single crystal it was found to be $4.07 \times 10^{5} \mathrm{~s}^{-1}\left(\tau_{S C, S R H}=2511 \mathrm{~ns}\right)$. The results agree well with earlier reports where the 
range of $k_{1}$ is from $10^{6}$ to $10^{9} s^{-1}{ }^{9}$. Furthermore, it is expected due to the better quality of SC over TF as $k_{1, S C}<k_{1, T F}$. Remarkably, doping density was estimated to be $1.38 \times 10^{8}$ to $2.01 \times 10^{13} \mathrm{~cm}^{-3}$ for SC and TF, respectively. Again, this is due to the better quality of SC than TF. The low concentration demonstrates the possible unintentional photodoping arising from acoustic phonon scattering ${ }^{56,57}$ or by trapped charge carriers from subgap states ${ }^{58}$. However, for accurate analysis, $k_{2} N_{D}$ should be compared with $k_{1}$ under low injection whereas under high injection the effect of $N_{0}$ also becomes significant.

Thin films exhibited higher band to band recombination, $k_{2}$, than single crystals $\left(k_{2, T F}=9.55 \times 10^{-12} \mathrm{~cm}^{3} / \mathrm{s}\right.$ and $k_{2, S C}=1.05 \times 10^{-13} \mathrm{~cm}^{3} / \mathrm{s}$. The increase in $k_{2}$ in thin films can be attributed to the presence of small grain boundaries which redshifts the bandgap of the material resulting in higher radiative recombination ${ }^{59,60}$. Manser and Kamat have reported a value of $2 \times 10^{-9} \mathrm{~cm}^{3} / \mathrm{s}$ for organometal halide perovskites ${ }^{61}$ while Johnston and Herz reported values ranging from $6 \times 10^{-11}$ to $9.2 \times 10^{-10} \mathrm{~cm}^{3} / \mathrm{s}$ for different hybrid perovskite materials ${ }^{9}$. The relatively higher $k_{2}$ in TF than $\mathrm{SC}$ is due to the additional localized radiative recombination from small grain boundaries ${ }^{59}$.

Table 1 Comparison of extracted parameters from TRPL for thin films and single crystal of perovskites.

\begin{tabular}{llll}
\hline Parameter & Unit & Thin Film & Single Crystal \\
\hline$k_{1}$ & $s^{-1}$ & $7.47 \times 10^{6}$ & $4.07 \times 10^{5}$ \\
\hline$k_{2}$ & $\mathrm{~cm}^{3} / s$ & $9.55 \times 10^{-12}$ & $1.05 \times 10^{-13}$ \\
\hline$N_{D}$ & $\mathrm{~cm}^{3}$ & $2.01 \times 10^{13}$ & $1.38 \times 10^{8}$ \\
\hline$\mu$ & $\mathrm{cm}^{2} / V s$ & 0.18 & 2.04 \\
\hline$S_{L}$ (Glass/Absorber) & $\mathrm{cm} / \mathrm{s}$ & 24.54 & 5.75 \\
\hline$S_{R}$ (Absorber) & $\mathrm{cm} / \mathrm{s}$ & $5.89 \times 10^{4}$ & 1.00 \\
\hline$S_{R}$ (Absorber/HTM) & $\mathrm{cm} / \mathrm{s}$ & $8.91 \times 10^{5}$ & - \\
\hline$P L E$ & - & $3.16 \times 10^{-5}$ & $1.62 \times 10^{-4}$ \\
\hline
\end{tabular}

Extraction of carrier mobility from TRPL transients resulted in values of $0.18 \mathrm{~cm}^{2} / \mathrm{Vs}$ for TF which was increased to $2.04 \mathrm{~cm}^{2} / \mathrm{Vs}$ for SC. For the same SC, minority carrier mobility was estimated as 10 by Wenger etal ${ }^{29}$. Similar mobilities have been reported by earlier works on SC such as $2.5 \mathrm{~cm}^{2} /$ Vs for PSC using spacecharge-limited current technique by Shi etal ${ }^{6,62}$. On the other hand, mobilities for thin film PSC covers a large span from $10^{-4}$ to $10 \mathrm{~cm}^{2} / \mathrm{Vs}^{63}$. The difference between the thin films and single crystals in PSC suggests that the grain morphology and boundaries is a huge factor in determining the carrier mobility in these materials. Herz ${ }^{62}$ has showed recently that there exists a wide range of charge carrier mobility arising due to experimental uncertainties from different experiments even for the same stoichiometric material. Although it is expected for the carrier mobilities for SC to be higher than TF, the difference is not found to be huge in the literature ${ }^{62}$.

PLE for the SC and TF was calculated to be $3.16 \times 10^{-5}$ and $1.62 \times 10^{-4}$, respectively. PLE in this order of $10^{-4}$ to $10^{-5}$ shows a good potential for PSC when compared with previous generation solar cells ${ }^{64}$. It is vital to understand that PL as a result of radiative recombination is dependent on the nature of the bandgap, direct or 
indirect. This could be observed from Green's consolidated list of both direct and indirect band semiconductors for PLE which has a huge span ranging from $2.25 \times 10^{-1}$ for GaAs to $2.7 \times 10^{-9}$ for OPV cell ${ }^{64}$. Even in the same direct band family, thin film with the localized grain boundaries may yield higher recombination ${ }^{65}$ as observed in PL results (Table 1) than its counterpart SC with higher crystallinity.

\section{Conclusion}

A comprehensive TRPL analysis has been carried out to assess the carrier dynamics due to bulk and interfacial recombination in perovskite films. The work herein provides insight into the transport and extraction kinetics by decoupling the effect of different processes contributing to TRPL signal. By linking this rate model with the experimental measurements, the purpose is to go beyond the conventional fitting life times and extract the physical parameters such as $S_{L}, S_{R}, k_{1}, k_{2}, \mu$ and $N_{D}$. An excellent conformity of fits to experimental data is found by including the effect of diffusion, doping density and surface recombination velocity at interfaces for accurate representation of TRPL kinetics. The resulted physical parameters from thin films and single crystals of perovskites clarified the distinct nature of both materials and provided hints for possible improvements. The PL signal quenching is found to be due to the efficient carrier transfer to the interface which included both the carrier extraction from the absorber to HTM and SRH recombination due to interfacial defects. Under low injection level and low doping concentration, the decay of the photogenerated carriers is mainly controlled by the SRH recombination rate, $k_{1}$, whereas radiative recombination, $R_{2}$, becomes significant under high injection level leading to higher PL efficiency. For accurate assessment and identification of the dominant recombination from PL decay, it is recommended to compare $k_{1}$ with $k_{2}\left(N_{D}+N_{0}\right)$. The ability of the proposed charge dynamics model to swiftly extract meaningful optoelectronic parameters from TRPL signal makes it a standard characterization tool for acquiring in-depth knowledge of the material.

\section{Acknowledgements}

The authors are grateful to Wenger et al ${ }^{29}$ for providing the TRPL data of perovskite single crystal film to validate the robustness of the developed method.

G. G. acknowledge support from the Swiss National Science Foundation (SNSF), funding through the Ambizione Energy project HYPER (grant number PZENP2_173641). 


\section{References}

1. Motta, C. et al. Revealing the role of organic cations in hybrid halide perovskite CH3NH3PbI3. Nat. Commun. 6, 7026 (2015).

2. Zhang, Y. et al. Direct-Indirect Nature of the Bandgap in Lead-Free Perovskite Nanocrystals. J. Phys. Chem. Lett. 8, 3173-3177 (2017).

3. Ke, X., Yan, J., Zhang, A., Zhang, B. \& Chen, Y. Optical band gap transition from direct to indirect induced by organic content of CH 3 NH 3 PbI 3 perovskite films. Appl. Phys. Lett. 107, 91904 (2015).

4. Rehman, W. et al. Charge-Carrier Dynamics and Mobilities in Formamidinium Lead Mixed-Halide Perovskites. Adv. Mater. 27, 7938-7944 (2015).

5. Zhumekenov, A. A. et al. Formamidinium Lead Halide Perovskite Crystals with Unprecedented Long Carrier Dynamics and Diffusion Length. ACS Energy Lett. 1, 32-37 (2016).

6. Shi, D. et al. Low trap-state density and long carrier diffusion in organolead trihalide perovskite single crystals. Science 347, 519-22 (2015).

7. Wehrenfennig, C., Liu, M., Snaith, H. J., Johnston, M. B. \& Herz, L. M. Charge-Carrier Dynamics in Vapour-Deposited Films of the Organolead Halide Perovskite CH3NH3PbI3- xClx. Energy Environ. Sci. 7, 2269 (2014).

8. Hutter, E. M., Eperon, G. E., Stranks, S. D. \& Savenije, T. J. Charge Carriers in Planar and MesoStructured Organic-Inorganic Perovskites: Mobilities, Lifetimes, and Concentrations of Trap States. $J$. Phys. Chem. Lett. 6, 3082-3090 (2015).

9. Johnston, M. B. \& Herz, L. M. Hybrid Perovskites for Photovoltaics: Charge-Carrier Recombination, Diffusion, and Radiative Efficiencies. Acc. Chem. Res. 49, 146-154 (2016).

10. Berdiyorov, G. R., El-Mellouhi, F., Madjet, M. E., Alharbi, F. H. \& Rashkeev, S. N. Electronic transport in organometallic perovskite $\mathrm{CH} 3 \mathrm{NH} 3 \mathrm{PbI} 3$ : The role of organic cation orientations. Appl. Phys. Lett. 108, 53901 (2016).

11. Berdiyorov, G. R. et al. Effect of halide-mixing on the electronic transport properties of organometallic perovskites. Sol. Energy Mater. Sol. Cells 148, 2-10 (2016).

12. Leijtens, T. et al. Carrier trapping and recombination: the role of defect physics in enhancing the open circuit voltage of metal halide perovskite solar cells. Energy Environ. Sci. 9, 3472-3481 (2016).

13. Wang, B., Wong, K. Y., Yang, S. \& Chen, T. Crystallinity and defect state engineering in organo-lead halide perovskite for high-efficiency solar cells. J. Mater. Chem. A 4, 3806-3812 (2016).

14. Augulis, R. et al. Multistep Photoluminescence Decay Reveals Dissociation of Geminate Charge Pairs in Organolead Trihalide Perovskites. Adv. Energy Mater. 7, (2017).

15. Chang, H. C. et al. Improvement on industrial n-type bifacial solar cell with $>20.6 \%$ efficiency. Energy Procedia 55, 643-648 (2014).

16. Milot, R. L. et al. Radiative Monomolecular Recombination Boosts Amplified Spontaneous Emission in HC(NH 2 ) 2 SnI 3 Perovskite Films. J. Phys. Chem. Lett. 7, 4178-4184 (2016).

17. Wetzelaer, G. J. A. H. et al. Trap-Assisted Non-Radiative Recombination in Organic-Inorganic Perovskite Solar Cells. Adv. Mater. 27, 1837-1841 (2015).

18. Baloch, A. A. B., Hossain, M. I., Tabet, N. \& Alharbi, F. H. Practical Efficiency Limit of Methylammonium Lead Iodide Perovskite (CH 3 NH 3 PbI 3 ) Solar Cells. J. Phys. Chem. Lett. 9, 426-434 (2018).

19. Baloch, A. A. B. et al. Full space device optimization for solar cells. Sci. Rep. 7, 11984 (2017).

20. Ye, M. et al. Recent advances in interfacial engineering of perovskite solar cells. J. Phys. D. Appl. Phys. 50, aa7cb0 (2017).

21. Chen, Q. et al. Under the spotlight: The organic-inorganic hybrid halide perovskite for optoelectronic applications. Nano Today 10, 355-396 (2015).

22. Webber, D. et al. Carrier diffusion in thin-film CH 3 NH 3 PbI 3 perovskite measured using four-wave mixing. Appl. Phys. Lett. 111, 121905 (2017).

23. Paulke, A. et al. Charge carrier recombination dynamics in perovskite and polymer solar cells. Appl. Phys. Lett. 108, (2016).

24. Grancini, G. et al. Femtosecond Charge-Injection Dynamics at Hybrid Perovskite Interfaces. ChemPhysChem 18, 2381-2389 (2017). 
25. Handa, T., Tex, D. M., Shimazaki, A., Wakamiya, A. \& Kanemitsu, Y. Charge Injection Mechanism at Heterointerfaces in CH3NH3PbI3 Perovskite Solar Cells Revealed by Simultaneous Time-Resolved Photoluminescence and Photocurrent Measurements. J. Phys. Chem. Lett. 8, 954-960 (2017).

26. Stranks, S. D. et al. Electron-Hole Diffusion Lengths Exceeding 1 Micrometer in an Organometal Trihalide Perovskite Absorber. Science (80-. ). 342, 341-344 (2013).

27. Marchioro, A. et al. Unravelling the mechanism of photoinduced charge transfer processes in lead iodide perovskite solar cells. Nat Phot. 8, 250-255 (2014).

28. de Quilettes, D. W. et al. Impact of microstructure on local carrier lifetime in perovskite solar cells. Science (80-. ). 348, 683-686 (2015).

29. Wenger, B. et al. Consolidation of the optoelectronic properties of $\mathrm{CH} 3 \mathrm{NH} 3 \mathrm{PbBr} 3$ perovskite single crystals. Nat. Commun. 8, 590 (2017).

30. Piatkowski, P. et al. Direct monitoring of ultrafast electron and hole dynamics in perovskite solar cells. Phys. Chem. Chem. Phys. 17, 14674-14684 (2015).

31. Staub, F. et al. Beyond Bulk Lifetimes: Insights into Lead Halide Perovskite Films from TimeResolved Photoluminescence. Phys. Rev. Appl. 6, 1-13 (2016).

32. Wehrenfennig, C., Eperon, G. E., Johnston, M. B., Snaith, H. J. \& Herz, L. M. High charge carrier mobilities and lifetimes in organolead trihalide perovskites. Adv. Mater. 26, 1584-1589 (2014).

33. Handa, T., Tex, D. M., Shimazaki, A., Wakamiya, A. \& Kanemitsu, Y. Charge Injection Mechanism at Heterointerfaces in CH3NH3PbI3 Perovskite Solar Cells Revealed by Simultaneous Time-Resolved Photoluminescence and Photocurrent Measurements. J. Phys. Chem. Lett. acs.jpclett.6b02847 (2017). doi:10.1021/acs.jpclett.6b02847

34. Shockley, W. \& Read, W. T. Statistics of the Recombinations of Holes and Electrons. Phys. Rev. 87, 835-842 (1952).

35. Tvingstedt, K. et al. Radiative efficiency of lead iodide based perovskite solar cells. Sci. Rep. 4, 6071 (2015).

36. Milot, R. L. et al. Radiative Monomolecular Recombination Boosts Amplified Spontaneous Emission in HC(NH2)2SnI3 Perovskite Films. J. Phys. Chem. Lett. 7, 4178-4184 (2016).

37. Hages, C. J. et al. Identifying the Real Minority Carrier Lifetime in Nonideal Semiconductors: A Case Study of Kesterite Materials. Adv. Energy Mater. 7, 1-10 (2017).

38. Wang, K. \& Kampwerth, H. Separation algorithm for bulk lifetime and surface recombination velocity of thick silicon wafers and bricks via time-resolved photoluminescence decay. J. Appl. Phys. 115, 173103 (2014).

39. Yang, Y. et al. Low surface recombination velocity in solution-grown $\mathrm{CH} 3 \mathrm{NH} 3 \mathrm{PbBr} 3$ perovskite single crystal. Nat. Commun. 6, 7961 (2015).

40. Dobrich, A., Schwarzburg, K. \& Hannappel, T. Interface analysis on MOVPE grown InP-GaInAs-InP double heterostructures for application in infrared solar cells. Sol. Energy Mater. Sol. Cells 148, 25-29 (2016).

41. Ahrenkiel, R. K. Measurement of minority-carrier lifetime by time-resolved photoluminescence. Solid. State. Electron. 35, 239-250 (1992).

42. Mattheis, J., Werner, J. H. \& Rau, U. Finite mobility effects on the radiative efficiency limit of pnjunction solar cells. Phys. Rev. B 77, 85203 (2008).

43. Davies, C. L. et al. Bimolecular recombination in methylammonium lead triiodide perovskite is an inverse absorption process. Nat. Commun. 9, 293 (2018).

44. Meier, D. L., Hwang, J. M. \& Campbell, R. B. The Effect of Doping Density and Injection Level on Minority-Carrier Lifetime as Applied to Bifacial Dendritic Web Silicon Solar Cells. IEEE Trans. Electron Devices 35, 70-79 (1988).

45. Wen, X. et al. Defect trapping states and charge carrier recombination in organic-inorganic halide perovskites. J. Mater. Chem. C 4, 793-800 (2016).

46. Ablekim, T. et al. Self-compensation in arsenic doping of CdTe. Sci. Rep. 7, 4563 (2017).

47. Connelly, B. C. et al. Dependence of minority carrier lifetime of Be-doped InAs/InAsSb type-II infrared superlattices on temperature and doping density. Phys. status solidi 253, 630-634 (2016).

48. Saba, M. et al. Correlated electron-hole plasma in organometal perovskites. Nat. Commun. 5, 5049 (2014).

49. Lin, Y. et al. $\pi$-Conjugated Lewis Base: Efficient Trap-Passivation and Charge-Extraction for Hybrid Perovskite Solar Cells. Adv. Mater. 29, 1604545 (2017). 
50. Wang, H.-H. et al. Improving the TiO 2 electron transport layer in perovskite solar cells using acetylacetonate-based additives. J. Mater. Chem. A 3, 9108-9115 (2015).

51. Yang, D. et al. High efficiency flexible perovskite solar cells using superior low temperature TiO 2. Energy Environ. Sci. 8, 3208-3214 (2015).

52. Xing, G. et al. Low-temperature solution-processed wavelength-tunable perovskites for lasing. Nat. Mater. 13, 476-480 (2014).

53. Beard, M. C., Turner, G. M. \& Schmuttenmaer, C. A. Transient photoconductivity in GaAs as measured by time-resolved terahertz spectroscopy. Phys. Rev. B 62, 15764-15777 (2000).

54. Sabbah, A. J. \& Riffe, D. M. Measurement of silicon surface recombination velocity using ultrafast pump-probe reflectivity in the near infrared. J. Appl. Phys. 88, 6954-6956 (2000).

55. Peng, W. et al. Influence of growth temperature on bulk and surface defects in hybrid lead halide perovskite films. Nanoscale 8, 1627-1634 (2016).

56. Mante, P.-A., Stoumpos, C. C., Kanatzidis, M. G. \& Yartsev, A. Electron-acoustic phonon coupling in single crystal $\mathrm{CH} 3 \mathrm{NH} 3 \mathrm{PbI} 3$ perovskites revealed by coherent acoustic phonons. Nat. Commun. 8, 14398 (2017).

57. Peng, W. et al. Ultralow Self-Doping in Two-dimensional Hybrid Perovskite Single Crystals. Nano Lett. 17, 4759-4767 (2017).

58. Stranks, S. D. et al. Recombination Kinetics in Organic-Inorganic Perovskites: Excitons, Free Charge, and Subgap States. Phys. Rev. Appl. 2, 1-8 (2014).

59. D'Innocenzo, V., Srimath Kandada, A. R., De Bastiani, M., Gandini, M. \& Petrozza, A. Tuning the Light Emission Properties by Band Gap Engineering in Hybrid Lead Halide Perovskite. J. Am. Chem. Soc. 136, 17730-17733 (2014).

60. Correa-Baena, J.-P. et al. Identifying and suppressing interfacial recombination to achieve high opencircuit voltage in perovskite solar cells. Energy Environ. Sci. 1, 556-560 (2017).

61. Manser, J. S. \& Kamat, P. V. Band filling with free charge carriers in organometal halide perovskites. Nat. Photonics 8, 737-743 (2014).

62. Herz, L. M. Charge-Carrier Mobilities in Metal Halide Perovskites: Fundamental Mechanisms and Limits. ACS Energy Lett. 2, 1539-1548 (2017).

63. Maynard, B. et al. Electron and hole drift mobility measurements on methylammonium lead iodide perovskite solar cells Electron and hole drift mobility measurements on methylammonium lead iodide perovskite solar cells. 173505, 2-6 (2016).

64. Green, M. A. Radiative efficiency of state-of-the-art photovoltaic cells. Prog. Photovoltaics Res. Appl. 20, 472-476 (2012).

65. Zhang, L. et al. Ultra-bright and highly efficient inorganic based perovskite light-emitting diodes. Nat. Commun. 8, 15640 (2017). 